Canadian University Music Review

Revue de musique des universités canadiennes

\title{
Une femme musicienne dans un monde masculin : la voix de Begum Akhtar
}

\section{Regula Burckhardt Qureshi}

Volume 16, numéro 1, 1995

Voices of Women: Essays in Honour of Violet Archer

Voix de femmes : mélanges offerts à Violet Archer

URI : https://id.erudit.org/iderudit/1014418ar

DOI : https://doi.org/10.7202/1014418ar

Aller au sommaire du numéro

\section{Éditeur(s)}

Canadian University Music Society / Société de musique des universités canadiennes

\section{ISSN}

0710-0353 (imprimé)

2291-2436 (numérique)

Découvrir la revue

Citer cet article

Burckhardt Qureshi, R. (1995). Une femme musicienne dans un monde masculin : la voix de Begum Akhtar. Canadian University Music Review / Revue de musique des universités canadiennes, 16(1), 96-113.

https://doi.org/10.7202/1014418ar

\section{Résumé de l'article}

L'article situe la vie de musicienne d'une grande chanteuse dont la carrière va du milieu féodal et colonial des cours et des salons jusqu'aux scènes fréquentées par la classe moyenne urbaine d'une Inde indépendante. On y retrouve la question habituellement problématique de l'identité sociale de cette musicienne héréditaire, qui est le corollaire inévitable de l'association entre hommes et interprètes, mais aussi la condition essentielle à

l'apprentissage et à la pratique de l'art de la musique. Comment Begum Akhtar a rapidement acquis une réputation en musique grâce à l'appui des nobles, comment elle est " passée » dans le monde respectable du mariage, et comment elle a redéfini son art et sa personne, émergeant comme l'idole nationale de la culture musicale de cour de l'Inde - c'est seulement dans les marges que l'on peut voir tous ces aspects, et ce par le biais d'un kaléidoscope de sources positionnées et de l'expression musicale stylisée d'une artiste dépendante des hommes mais qui a aussi créé des liens musicaux avec des femmes (incluant l'auteure).
All Rights Reserved (C Canadian University Music Society / Société de musique des universités canadiennes, 1995
Ce document est protégé par la loi sur le droit d'auteur. L’utilisation des services d'Érudit (y compris la reproduction) est assujettie à sa politique d'utilisation que vous pouvez consulter en ligne.

https://apropos.erudit.org/fr/usagers/politique-dutilisation/ 


\section{UNE FEMME MUSICIENNE DANS UN MONDE MASCULIN : LA VOIX DE BEGUM AKHTAR}

\section{Regula Burckhardt Qureshi}

Begum Akhtar demeure un nom bien connu en Inde d' aujourd'hui ${ }^{1}$. Même une vingtaine d'années après son décès, l'art musical de cette femme continue à être diffusé et apprécié grâce aux nombreux enregistrements réalisés au cours sa carrière de chanteuse qui s'est étalée sur plus de 40 ans. On garde le souvenir de cette dame dont l'art a triomphé à la fois sous le patronage de la cour, dans les salons féodaux, plus tard sur la scène théâtrale et dans les films aussi bien que dans la salle de concert nationale et internationale, après l'indépendance de l'Inde. On admire aussi la transformation personnelle de cette chanteuse courtisane en une épouse respectable qui ira jusqu'à abandonner sa carrière musicale et à ressurgir à nouveau le symbole national de la culture qui l'avait façonnée. Begum Akhtar continue de vivre dans le cœur de ses auditeurs car, selon le célèbre poète Faiz Ahmad Faiz, " son charme personnel, sa grande générosité de cœur et sa sympathie pour les bonnes causes la rendaient toute aussi attrayante que son art lui-même $»^{2}$.

En Inde, tout comme en Occident, la littérature musicale dit peu de choses au sujet des femmes. Begum Akhtar est sans contredit l'une de ces femmes sur lesquelles on a le plus écrit, ne serait-ce que sous la forme de quelques brèves biographies. Dans l'ensemble ces courts récits nous fournissent des informations fondamentales sur sa vie et les hauts faits de sa carrière tout en offrant une certaine appréciation de sa musique et de sa personnalité. Mais ils touchent à peine au milieu socio-musical dans lequel une femme musicienne d'alors devait fonctionner. Si l'on veut saisir jusqu'à quel point la vie de Begum Akhtar a été remarquable, il faut rétablir le lien entre son histoire et ce contexte, tout en se référant aux changements importants qui ont transformé la vie musicale de son temps. Je me propose donc, dans ce travail préliminaire à un collage biographique, d'esquisser ce contexte qui, il faut le dire, offre en dernière analyse peu d'information et ceci pour des raisons qui deviendront apparentes lorsqu'on examinera justement le contexte en question. Comme sources d'information, j'ai puisé non seulement dans les écrits existants, mais aussi dans les témoi-

1 Je désire exprimer ma profonde renconnaissance à Albert La France et à Nicole Beaudry pour leur traduction sensible et nuancée de mon texte anglais ; mes sincères remerciments aussi à M. Umar Ansari, à $M$. Arvind Parikh, à M. Abdul Qavi Zia et aux regrettés $M^{\text {me }}$ Naina Devi et $M$. G. N. Joshi pour leurs précieux souvenirs de Begum Akhtar qu'ils ont partagés avec moi.

2Faiz Ahmad Faiz ; voir le texte du disque Begum Akhtar in Memoriam : Kalam-e-Asatiza (1975). Les notices complètes des enregistrements sont données en appendice dans l'ordre chronologique d'enregistrement. 
gnages de gens qui ont connu et apprécié Begum Akhtar à différentes étapes de sa vie. Si j'ose me compter marginalement parmi ces personnes, c'est que mon désir d'écrire au sujet de cette merveilleuse artiste remonte à trois occasions inoubliables où j'ai eu le privilège d'entrer en contact avec elle et son art, il y plus de 25 ans.

Le succès qu'a connu la carrière de Begum Akhtar illustre clairement l'impact que le raffinement poétique a pu avoir sur la grandeur musicale et personnelle de cette dame. Durant la dernière décennie de sa vie, sa musique « légère » faisait partie des concerts les plus prestigieux de musique classique ; envoyée dans différents pays pour représenter la culture musicale indienne, elle s'est vue conférer par le gouvernement de l'Inde les plus grandes distinctions culturelles. Si elle a continué de chanter d'autres genres de chansons musicalement plus « classiques » et basées sur le raga, tel le thumri et le dadra, son identité musicale est devenu totalement liée au ghazal de Urdu, d'où son titre populaire de « Reine du Ghazal (Malika-e-Ghazal) ».

En tant que langage culturel, la poésie a fait partie de la vie personnelle de Begum Akhtar et de son époux, qui appartenait à l'élite de Lucknow. Celui-ci, après le décès de son épouse, a fait publiquement son éloge dans des vers urdu. Sur un plan plus personnel, elle nous a laissé avant de mourir, à mon époux et à moi, un adieu dans les vers suivants :

Si tu te souviens de moi, je suis alors près de ton cœur ;

Mais si tu m'oublies, alors nous nous sommes éloignés ${ }^{3}$.

Certes, on se souvient de Begum Akhtar, mais certains se souviennent aussi qu' après son décès, lorsque le train rapportait sa dépouille mortelle à Lucknow, personne n'était là pour la réclamer, ni son mari, un avocat éminent, ni quelqu'un de sa famille courtisane, comme si elle était une inconnue.

Que nous reste-t-il alors de la vie musicale de Begum Akhtar ? Après avoir rassemblé tous les souvenirs, il nous reste un curieux sentiment d'absence et d'ambivalence devant la remarquable carrière artistique et personnelle de cette dame. Absence parce qu'on n'y retrouve point les contraintes et les appuis traditionnels caractéristiques d'un ordre socio-musical entièrement patriarcal. Ambivalence car si, d'une part, sa carrière porte les marques de son inévitable dépendance des protagonistes masculins issus de l'ordre patriarcal, d'autre part, elle fait preuve d'une émancipation personnelle vis-à-vis des pouvoirs masculins.

L'ordre patriarcal n'a pas empêché que des voix éminentes, voire affectives, parlent de cette femme. Mais qui peut être son avocate, qui peut parler en son nom ? À l'exception de certaines marques de solidarité de la part de quelques pairs, la communauté féminine de l'époque garde le silence, de même que les collègues et les disciples qui normalement auraient apporté leurs perspectives éloquentes sur sa musique, comme ils l'auraient fait pour un grand musicien

3Écrit à la main sur le disque Begum Akhtar Sings Ghalib : A Ghalib Centenary Production (1968) et dont l'artiste nous a fait cadeau en 1969. 
classique. Ceux qui se sont prononcés le plus clairement à son sujet sont des hommes qui valorisaient les interprétations que livrait Begum Akhtar dans les salons féodaux. Ces hommes, toutefois, traitaient de l'aspect circonstanciel de l'interprétation plutôt que de la dimension structurale de la musique.

En dépit de tout ceci, la voix de sa musique s'exprime clairement, avec force et individualisme, et avec une riche complexité qui témoignent de l'expérience d'une vie personnelle, musicale et surtout humaine, des plus remarquables et des plus dynamiques. Ce qui suit alors est une tentative de cerner les épisodes importants de cette vie en relation avec les contextes changeants que j'ai mentionnés plus haut. Je m'appliquerai à montrer comment, en tant que femme, Begum Akhtar s'est conduite à l'égard des hommes qui ont défini et contrôlé ces contextes, et comment ses relations avec eux se sont manifestées dans sa musique.

Une biographie est constituée nécessairement à partir de différentes voix, chacune exprimant son propre point de vue, visant son propre auditoire et représentant ses propres intérêts. Ces voix multiples façonnent celle de l'artiste et sont à leur tour absorbées par celle de l'auteur. La fragilité d'une telle démarche devient particulièrement évidente dans le cas d'une artiste dont le statut de symbole national se heurte à son identité sociale contestée. Que l'on veuille effacer ou reconnaître cette identité, le fait demeure que la vie et la musique de Begum Akhtar nous parviennent par l'entremise de divers participants, des hommes pour la plupart, qui l'ont connue par sa musique : son époux, ses bienfaiteurs, ses amis, des musiciens, des poètes. Parmi les trois écrivains qui parlent de Begum Akhtar se distinque Susheela Misra, qui lui consacre quelques chapitres biographiques dans lesquels elle incorpore, mais sans bien les identifier, des renseignements sur sa jeunesse, fournis par l'artiste ellemême.

La voix de Begum Akhtar, en plus de l'éloquence de sa musique, nous parvient fortuitement de poèmes très courts, sous forme de dédicace, semblables à ceux qui sont cités plus haut. D'une plus grande importance est l'image que Begum Akhtar elle-même nous laisse dans de brèves présentations des chansons qu'elle a enregistrées. Ses paroles nous communiquent deux messages importants : d'abord comment elle s'identifie à la poésie et ensuite comment elle se dévoue pour ses auditeurs ${ }^{4}$. Ce qui suit alors est une tentative de recréer le contexte biographique qui a donné naissance à cette image.

Née à Faizabad, une petite ville près de Lucknow, Begum Akhtar (1914 [ou 1910]-74) a grandi avec sa mère, une chanteuse professionnelle qui menait une existence difficile. Dès son jeune âge, la riche résonnance de sa voix et une remarquable mémoire musicale indiquaient qu'il lui fallait une éducation musicale avancée. Elle a donc étudié avec des maîtres professionnels qui se sont tous intéressés à gérer sa jeune carrière. Grâce à de nombreux déménagements, toujours dans des centres urbains plus importants, elle a reçu une formation musicale de trois professeurs. À Patna, il y avait Imdad Khan, un joueur de

4Begum Akhtar Talks About, and Sings, the Ghazal (1975). Le disque comprend une présentation de chaque ghazal par Begum Akhtar. 
sarangi ${ }^{5}$, bien connu pour ses accompagnements de célèbres chanteuses courtisanes. Avec lui, Akhtari (un diminutif de Akhtar typiquement employé par les courtisanes) a " éduqué sa voix », bien qu'elle ait jugé « trop difficile » le premier raga qu'elle devait apprendre. Puis à Gaya, Ghulam Muhammad Khan lui a enseigné brièvement. Mais le jeune frère de ce dernier, Atsa Muhammad Khan, est devenu son maître principal et son guide. Il a déménagé à Faizabad afin de poursuivre l'éducation de la jeune fille. Lorsque Akhtari a eu treize ans, il l'a fait déménager avec sa mère à Calcutta, la métropole de la musique, du théâtre, des studios d'enregistrement et du film à cette époque ${ }^{6}$.

L'entraînement d'Akhtari a suivi le cours du chanteur classique masculin, y compris plusieurs heures d'exercices quotidiens commençant par le registre grave (kharaj bharna) et faisant appel à des vocalises fort recherchées. Or, comme Ata Muhammad, musicien de talents divers, chantait aussi des mélodies touchantes, il lui a enseigné les sortes de chansons que les femmes présentaient en public. Cet enseignement a surtout fourni à la jeune fille un régime d'entrâ̂nement rigoureux qu'elle trouvait oppressif et ennuyeux mais que plus tard, comme artiste mûre, elle est en venu à apprécier. Elle exprimait une profonde reconnaissance envers son ustad de la manière suivante : "Aujourd'hui mon cœur est rempli de gratitude envers mon ustad qui a si patiemment formé ma voix et l'a rendue si flexible. ${ }^{7}$ »

Begum Akhtar reconnaît également avoir appris d'artistes féminines qu'elle a entendu à Calcutta. Aux noms des célèbres chanteuses courtisanes Jaddan Bai et Gauhar Jan, elle ajoute celui de Chandabai dont le chant l'avait impressionnée dans sa jeunesse à Faizabad. Parmi les chanteurs masculins de musique légère, elle avoue avoir été « inspirée " par un chanteur de thumri unique en son genre, Moizuddin Khan, ainsi que par Jhande Khan, célèbre pour ses chansons sur scène ${ }^{8}$.

L'autre aspect de sa formation avec Ata Muhammad Khan réside dans la gestion de sa carrière. C'est lui qui, sans doute, l'a initialement fait chanter dans des soirées privées et a négocié par la suite d'autres engagements pour elle. Mais c'est à l'occasion d'un concert public au bénéfice des victimes du tremblement de terre de Bihar, en 1934, alors que son professeur avait réussi à la présenter en l'absence d'un musicien plus âgé, que le public l'a acclamé pour la première fois. Sa prestation lui a mérité des critiques très favorables et les compliments personnels d'une importante politicienne nationale qui était présente au concert ${ }^{9}$.

5Le sarangi, qui est un instrument à archet, fournit l'accompagnement hétérophonique du chant dans la musique traditionnelle.

6 Susheela Misra, Music Makers of the Bhatkhande College (Calcutta : Sangeet Research Academy, 1985), 76 ; voir aussi Misra, Great Masters of Hindustan Music (New Delhi : Hem Publishers, 1981), 176-77; et Amal Das Sharma, Musicians of India Past and Present : Gharanas of Hindustani Music and Genealogies (Calcutta : Naya Prokash, 1993), 144.

7 Cité dans Misra, Great Masters of Hindustan Music, 177.

8 Misra, Great Masters of Hindustan Music, 176 ; idem, Music Makers of the Bhatkhande College, 76-77. Le thumri est le genre principal de la musique légère ou semi-classique.

9Misra, Music Makers of the Bhatkhande College, 76. 
C'est à cette époque, vers 1930, que Akhtari a été invitée à enregistrer une série de chansons semi-classiques variées chez HMV dont le siège était alors à Calcutta. Diffusés par la radio et dans des établissements commerciaux désireux d'attirer de la clientèle, tels les companies de thé, plusieurs de ces 78 tours ont connu un succès qui dure encore aujourd'hui. Le nom d'Akhtari est devenu très connu en raison de la coutume chez HMV à l'époque, qui voulait que les chanteuses se nomment à la fin d'un disque. "Mon nom est Akhtari Bai de Faizabad » disait-elle; ce célèbre signal contient le suffixe « bai » (dame) qui servait traditionnellement à identifier une chanteuse courtisane. Aujourd'hui, ses enregistrements font partie de son héritage, mais à l'époque ils n'apportaient que peu de récompense matérielle. Toutefois, c'est bien grâce à ces enregistrements que sa réputation a été établie si tôt dans sa carrière, ce qui l'a conduit sans doute vers un patronage plus étendu.

Calcutta était aussi, avant Bombay, le centre de la production cinématographique grandissante de l'Inde. Tout comme le théâtre florissant, les compagnies de films produisaient des drames truffés de chansons et, en tant que chanteuse-actrice, Akhtari a participé à ces deux modes de production. Belle femme qu'elle était, elle est bientôt devenue la vedette d'un certain nombre de films d'envergure vers la fin des années 30 et au début des années 40, comme l'ont d'ailleurs fait d'autres éminentes chanteuses de musique semi-classique de l'époque ${ }^{10}$. En effet, en raison de ce médium populaire de chansons de film, Akhtari s'est mise à négliger la stricte discipline qu'exigeait la musique classique. Comme elle le racontait plus tard, ceci lui a mérité un ultimatum de la part de son professeur : "s soit que tu concentres sur ta musique ou que tu deviennes comédienne ${ }^{11}$ ! »

Mais c'était pour Ata Muhammad Khan l'occasion de mettre en cause plus que la qualité de la musique : sous sa tutelle, Akhtari Bai avait été formée en vue du patronage féodal, un procédé dans lequel il devait continuer de lui servir de guide et d'accompagnateur. C'est ainsi que, durant cette même période à Calcutta, Akhtari s'est mise à accepter de l'emploi dans les établissements féodaux. Son premier engagement important était au service du Raja de Nandgaon qui a l'invité, avec sa mère, à résider dans sa demeure. Sa célébrité croissante lui a apporté des invitations des principales cours indiennes, y compris celle du Maharaja du Cachemire et celle du Nawab de Rampour chez qui elle a demeuré pendant quelque temps, et qui l'a récompensée royalement ${ }^{12}$. On connaît mal quelle a pu être la nature personnelle et musicale de ces épisodes au sein d'une élite en milieu privé. Il est clair, cependant, que Akhtari Bai circulait avec assurance dans des cercles de connaisseurs riches et cultivés parmi lesquels se trouvait d'ailleurs son futur mari, un bon ami du Nawab de Ram-

10 Ceci était avant la technique du «playback », qui permet de remplacer la voix de l'actrice par celle de la chanteuse ; voir aussi Allison Arnold, « Popular Film Song in India : A Case of Mass-Market Musical Eclecticism », dans Popular Music in India (Delhi : Manohar, 1988), 177-88.

11 Misra, Great Masters of Hindustan Music, 178.

12 Umar Ansari, conversation avec Regula B. Qureshi (Lucknow, 1992) ; Naina Devi, conversations avec Regula B. Qureshi (Delhi, 1987, 1992) ; et Abdul Qavi Zia, conversation avec Regula B. Qureshi (Edmonton, Alberta, 1992). 
pour ${ }^{13}$. En conséquence, son art musical déjà si expressif et cultivé s'est enrichi de sensibilité et de raffinement poétique et lui permettait de s'exprimer, lors de ses propres récitals, avec plus de profondeur et de sensibilité. De plus, sur le plan personnel, venait s'ajouter à son style d'interaction personnelle si plein d'esprit, une aisance de réplique et de citations poétiques tant prisée chez les élites de la langue ourdue.

Bombay était un autre centre important de patronage et d'emploi musicale pour Akhtari Bai. C'est ici qu'elle a enregistré plusieurs chansons de film en recourant à la technique du « playback » et qu'elle s'exécutait devant de riches mécènes. On compte parmi ceux-ci, tout particulièrement, l'homme d'affaires et poète Yahya Jasdanwalla ${ }^{14}$, ainsi que Zahir Yar Jung, un ministre de la cour d'Hyderabad qui, lors de ses visites à Bombay, la recevait en récital dans son salon-sur-rails ${ }^{15}$.

Vers la fin des années 30, grâce aux moyens financiers qu'elle avait accumulés, la renommée Akhtari Bai pouvait maintenant s'établir à Lucknow à titre d'artiste célèbre et être recherchée par les bienfaiteurs raffinés des centres de culture courtoise. Elle a fait l'acquisition de sa propre maison, située dans un bon voisinage, et l'a nommé « Villa Akhtar » (Akhtar Manzil) à la manière des gens de marque. C'est ici que sa mère et elle accueillaient invités et bienfaiteurs. Elle chantait également lors d'occasions spéciales ; le mariage en était une, car dans cette partie de l'Inde, la célébration d'un mariage n'était pas complète à moins qu'une chanson et une danse ne soient exécutées par une femme. Plus d'un demi-siècle plus tard, en 1938, Umar Ansari se souvient comment Akhtari avait ravi tout son auditoire à l'occasion du mariage de la fille d'un riche entrepreneur. Suivant la tradition courtisane, elle a non seulement chanté mais aussi précédé sa chanson d'une danse sur une célèbre chanson d'amour hindi, fredonnant et mimant toute une myriade d'émotions contenues dans ce simple vers : «Oiseau, cesse de chanter le nom de mon bien-aimé ${ }^{16}$. "

Mais plus que toutes autres choses, Akhtari était l'interprète musicale de poèmes ghazals la plus sollicitée de ce centre incontesté de la poésie ourdoue. Lors du festival annuel pan-indien de poésie nouvelle et de musique de Lucknow, elle avait l'honneur incontestable de présenter, année après année, les vers du plus célèbre poète, et ce au moment le plus convoité du programme, c'est-à-dire à la toute fin ${ }^{17}$. La célébrité de son chant rendait également célèbre le nouveau poème. Lors de ces occasions formelles Akhtari s'habillait à la manière traditionnelle de la cour : elle portait un corsage, une jupe ample et un pantalon étroit. Mais elle était aussi une femme moderne qui portait des panta-

13 Ansari, conversation avec Regula B. Qureshi (Lucknow, 1994).

14Jasdanwalla a conservé sur disque quelques enregistrements de Begum Akhtar en concert et qui nous donnent un précieux aperçu de son style ; voir le disque Malika-E-Ghazal Begum Akhtar in Mehfil (1983).

15G. N. Joshi, Down Melody Lane (Hyderabad : Orient Longman, 1984), 69-70, 132-33.

16En hindi, la ressemblance onomatopéique entre l'oiseau (papiha), son cri (piu) et le terme bien-aimé (pia) enrichit l'aspect affectif de ce vers.

$17 \mathrm{Zia}$, conversation avec Regula B. Qureshi (Edmonton, Alberta, 1992); et Ansari, conversation avec Regula B. Qureshi (Lucknow, 1992). 
lons, fumait et conduisait une Packard unique en son genre, un cadeau du Nawab de Rampour ${ }^{18}$. Elle projetait l'image d'une «splendeur taillée sur mesure » et d'une " brillante personnalité », d'une " beauté au teint clair », pleine de sourire, de charme et d'affection. Nul ne s'étonne que le souvenir de ses récitals et de sa brillante présence dans une Lucknow pré-indépendance soit si empreinte de nostalgie, de plaisir et d'admiration.

La période dans la vie de Begum Akhtar qu'une féministe du début du siècle désignait comme "celle de la seule véritable liberté pour les femmes (indiennes) ${ }^{19}{ }^{\prime}$ 'est achevé brusquement par le désir d'Akhtari elle-même. Tandis qu'à Lucknow la culture des exécutions musicales par des courtisanes connaissait un plein essor, elle profitait justement de son émancipation pour choisir l'état de dépendance qu'allait lui apporter son statut matrimonial. À en croire ce que l'on rapporte et selon les souvenirs de son mari, c'est elle qui, renversant toute convention établie, a demandé en mariage l'avocat Ishtiag Abbasi, un connaisseur prospère de Lucknow ${ }^{20}$.

En dépit d'une amitié préalable et de l'amour chez l'un et l'autre pour la poésie et la musique, le mariage a apporté des changements radicaux tant à son identité personnelle qu'à son mode de vie. Akhtari, la jeune fille, est devenue Akhtar Abbasi et le titre respectable «Begum » (Madame, Dame) a remplacé l'épithète courtisane "Bai ». En entrant dans la sphère domestique d'une maisonnée patrilinéaire respectable elle a cessé de donner des récitals et abandonné complètement le chant. En tant que Begum elle pouvait être l'hôtesse de soirées musicales chez elle, mais la parenté, les serviteurs et les amis ne devaient jamais, même en privé, entendre ce qu'elle avait été auparavant.

J'ai eu moi-même l'occasion de vivre l'effet de cette règle tacite alors que mon mari et moi étions reçus à dîner chez Begum Akhtar en 1969, plusieurs années après qu'elle avait repris sa brillante carrière publique. Comme elle me l'avait demandé, j'avais apporté mon sarangi, prévoyant qu'elle allait chanter et que j'allais jouer, même si je n'étais qu'une débutante. Par timidité et admiration pour elle, j'ai néanmoins laissé l'instrument dans la voiture et, lorsqu'elle m'a demandé d'apporter l'instrument dans la maison, j'ai avoué mon incompétence, tout en m'attendant à une seconde requête comme il est de mise dans cette culture lorsqu'il y a une si grande différence d'âge et de statut. Mais la seconde demande n'est pas venue et nous n'avons pas fait pas de musique, ce que j'ai toujours infiniment regretté. Hélas, je ne m'étais pas rendu compte à ce moment-là qu'en m'invitant, moi, une musicienne mariée et respectable, cette grande artiste m'invitait aussi à partager sa situation domestique et à briser un tabou qu'elle ne se sentait pas capable de briser seule et, que moi, comme étrangère j'aurais pu briser en faisant de la musique avec elle. Si seulement je l'avais su. Je n'avais pas réalisé non plus que, depuis son mariage, elle n'avait pas donné de concert à Lucknow ; elle ne le ferait qu'une seule fois avant sa

18 Ansari, conversation avec Regula B. Qureshi (Lucknow, 1992).

19Elizabeth Cooper, The Harim and the Purdah : Studies of Oriental Women (Delhi : Bimla Publishing House, 1915; réimpression, 1983), 89.

20 Ishtiaq Ahmed Abbasi, "Reflections and Fond Memories of Begum Akhtar 》, texte du disque Begum Akhtar Talks About, and Sings, the Ghazal (1975). 
mort, lors d'un concert au bénéfice de la défense nationale, et ceci devant un auditoire exclusivement féminin ${ }^{21}$.

Les habitudes personnelles de Begum Akhtar étaient également soumises à des contraintes au sein d'un foyer dominé par une sévère belle-sœur aînée. Lors d'une autre visite que je lui ai rendue, toujours en 1969, en plein jour cette fois, elle nous a entraînés, mon mari et moi, dans sa chambre à coucher afin d'avoir plus d'intimité et de pouvoir s'adonner à sa vieille habitude de fumer ; là, assises sur son lit, nous parlions de musique avec une cordialité toute simple, sans gêne ni contrainte.

Pourquoi Begum Akhtar s'est-elle servi de son indépendance pour devenir dépendante, et pourquoi à ce moment précis dans sa vie ? L'explication qu'elle donnait, telle que l'a rapporté son mari, était d'ordre personnel : elle désirait devenir l'épouse de celui qu'elle aimait. Dans un couplet finement tourné, dédié à son futur époux, elle déclare :

On peut aimer une bonne personne tant que l'on veut ;

Mais lorsqu'on est aimé en retour, on ne désire rien de plus ${ }^{22}$.

On ne saura jamais si d'autres facteurs sont entrés en ligne de compte : est-il possible, par exemple, qu'elle craignait l'interdit que voulait imposer le ministre de l'Information cette année-là, à l'effet qu'aucune femme musicienne professionnelle (une femme non respectable, donc) pourrait paraître sur les ondes de l'AIR (All India Radio) ? Pourtant, même si, par son mariage, elle était devenue une femme respectable, elle avait acceptée cet interdit qui découlait de son mariage. Par ailleurs, ce qui ressort clairement de l'histoire personnelle de Begum Akhtar, c'est une affirmation constante de son identité patrimoniale.

Dans sa présentation de vive voix à une série d'enregistrements de ghazals mis sur le marché après sa mort, Begum Akhtar a déclaré que son père poète l'exhortait à chanter de la poésie, spécialement les ghazals, et non pas n'importe quelle chanson. Ce geste et sa déclaration qu'elle n'était pas d'une famille de musiciens ${ }^{23}$, suggère qu'elle voulait nier ou du moins déguiser la stigmatisation du milieu de chanteurs professionnels. En outre, les poètes les plus âgés et les bienfaiteurs les plus importants s'entendent pour dire que le poète de Lucknow, Hakim Natiq, était le père d'Akhtar. Umar Ansari raconte comment, lors d'un festival de poésie et de musique, on avait demandé à Akhtar de chanter un poème de son père plutôt que celui d'un poète plus célèbre, puisque son père était présent au festival cette année-là ${ }^{24}$. Tout en admettant qu'elle ne pouvait avoir un père au sens social du terme, Umar Ansari note la relation quasi-familiale qui existait entre Hakim Natiq et Akhtari, et entre lui et la mère d'Akhtari qui

21 Parveen Talha, « Tawaefs : Lucknow's Colourful Ladies », The India Magazine 3, $\mathrm{n}^{0} 5$ (1983) : $62-67$.

22 Abbasi, lettre, «Reflections and Fond Memories of Begum Akhtar »; voir aussi Rekha Surya, «Ammi », The Illustrated Weekly of India 16, $\mathrm{n}^{\circ} 17$ (1991) : 46-47.

23 Voir le texte du disque Begum Akhtar Talks About, and Sings, the Ghazal (1975) ; Misra, Great Masters of Hindustan, 176.

24Ansari, conversation avec Regula B. Qureshi (Lucknow, 1992). 
le considérait comme son époux. De plus, les gens du voisinage considéraient Akhtar comme étant sa fille puisque, durant la longue maladie de Hakim Natiq, elle allait le voir tous les jours, jusqu'à sa mort ${ }^{25}$.

Mais la famille légitime du père de Begum Akhtar n'a jamais reconnu que celui-ci pouvait avoir des liens d'une part avec des courtisanes et, d'autre part, par hérédité, avec des musiciens professionnels, tous de réputation douteuse. De même, la famille de son mari n'entretenait aucune relation avec sa famille maternelle et le milieu qu'elle représentait ; Begum Akhtar elle-même ne leur rendait visite qu'en privé. De plus, en évitant de donner des concerts dans son milieu résidentiel, elle évitait tout contact professionnel avec des musiciens. Elle conservait ainsi une identité sociale respectable et pouvait continuer de le faire même après avoir recommencé à donner des concerts ailleurs. Lorsque son mari organisait des soirées musicales dans leur maison, elle y participait seulement en tant qu'auditrice ${ }^{26}$. Selon un ami avocat de son mari, elle entonnait parfois une chanson, en compagnie intime, mais jamais avec un accompagnement instrumental ${ }^{27}$.

Le retour de Begum Akhtar à sa carrière de chant lui a été suggéré, comme d'ailleurs toutes ses autres décisions musicales, par des hommes et motivé par des raisons personnelles et musicales. À peine cinq ans après son mariage, la mort de sa mère l'a laissée dans un état de désolation tellement grave que son mari a consenti à la laisser chanter, espérant qu'elle retrouverait ainsi son équilibre. Un réalisateur d'émissions musicales à l'All India Radio, qui connaissait la famille Akhtar, a encouragé Begum Akhtar et, bientôt, une première session d'enregistrement, chargée d'émotion, a été réalisée dans l'intimité du studio $^{28}$. Grâce à l'anonymat de l'enregistrement, elle pouvait, tout en conservant le respect dû à une femme mariée, recommencer à participer à des émissions radiophoniques régulières. Elle a continué donc de diffuser ses émissions à partir de Lucknow, et ensuite à l'échelle nationale à partir de Delhi, contribuant ainsi au mandat que se donnait la radio de disséminer la culture nationale. Selon le système universel d'évaluation de l'excellence musicale, chez l'AIR, elle a obtenu la classe $A$, la cote la plus élevée attribuable aux chanteurs de musique classique légère ${ }^{29}$.

C'est aussi à ce moment-là que Begum Akhtar a été réintroduite à la vie musicale toujours croissante d'une Inde indépendante dans laquelle les concerts étaient progressivement mieux soutenus par les institutions publiques, les élites gouvernementales et industrielles de même que par les associations musicales de la classe moyenne. Dépassant sa renommée antérieure, elle est devenue une

25Selon une voisine de Natiq, $M^{\text {me }}$ Siddiqa Qureshi, la voiture de Begum Akhtar annonçait inévitablement au voisinage la visite de la chanteuse ; Siddiqa Qureshi, conversation avec Regula B. Qureshi (Lucknow, 1992).

26Misra, Great Masters of Hindustan Music, 178.

27Zia, conversation avec Regula B. Qureshi (Sudbury, Ontario, 1994).

28 Uma Vasudev, « Ghazal Queen », Patriot Magazine (New Delhi) 10 novembre 1974 ; et Misra, Great Masters of Hindustani Music, 178-79.

29Misra, Great Masters of Hindustan Music, 179. La catégorie supérieure, « top class », était réservée aux artistes de la musique classique ; voir Daniel M. Neuman, The Life of Music in North India (Detroit : Wayne State University Press, 1990), 177, 178. 
artiste de réputation nationale dont la musique représentait la haute culture de Lucknow, un des centres nationaux qui reflétaient la gloire féodale. Durant les années 60 , elle a participé à plusieurs délégations culturelles à l'étranger : au Pakistan où ses chants suggéraient fortement la réconciliation, en Afghanistan où le roi l'a récompensée royalement, et finalement en URSS. Elle a reçu de son gouvernement plusieurs distinctions destinées à honorer une artiste de renommée nationale. Cette reconnaissance officielle ne fera qu'accroître son statut durant la dernière décennie de sa vie. D'ailleurs, depuis 1952, Begum Akhtar était redevenue une artiste célèbre par ses enregistrements, mais cette fois chez HMV à Bombay.

Cette reprise de sa carrière était en quelque sorte la continuation de la vie artistique de Begum Akhtar : les chansons qu'elle chantait et continuait d'ajouter à son répertoire étaient puisées dans la même source créatrice qui l'avait consacrée comme chanteuse célèbre avant son mariage. Les textes écrits à son sujet, aussi bien que ses disques, démontrent bien qu'elle chantait un mélange de genres de musique classique légère en hindi et en ourdou, pour lesquels elle puisait dans les formes traditionnelles conventionnelles et dans les ragas classiques. Comme auparavant, elle chantait et enregistrait sur disque les compositions des autres ${ }^{30}$, mais elle composait de plus en plus sa propre musique sur des poèmes de son choix ${ }^{31}$.

Toutefois, les ghazals en ourdou continuaient d'être la spécialité de Begum Akhtar comme à ses premières années à Lucknow. Les poètes la connaissaient, l'honoraient et la protégeaient puisque ses interprétations mettaient constamment en valeur l'œuvre des poètes contemporains. En 1968 elle a été tout naturellement choisie pour interpréter et enregistrer les œuvres de Ghalib, le poète le plus remarquable de la littérature ourdoue, à l'occasion de son centenaire. Elle a aussi continué de transformer les versets de ses chants en un langage personnel, comme elle avait été entraînée de le faire dans ce style de présentation hautement interactive de sa profession. Les auditeurs se souviennent comment, au Pakistan, elle a traité la douloureuse question du conflit entre les deux pays avec une chanson d'amour hindi, Viens, mon amour, cessons notre querelle. Au cours d'un de ses derniers récitals, alors qu'elle souffrait de la maladie qui devait l'emporter, elle chantait ce célèbre ghazal urdu :

Le nombre de vos amants ne sera pas moindre,

Sauf que moi, je ne serai plus parmi eux ${ }^{32}$.

Le développement musical de Begum Akhtar a aussi connu un changement, ou plutôt une transformation importante, car elle en est venue à adapter ses présentations au contexte du récital sur scène (plutôt que dans un salon) et à tenir compte de toutes les exigences propres à l'exécution de la musique

30Voir les textes des disques Begum Akhtar : Ghazals (1971) et Lost Horizons : Begum AkhtarGhazals (1977).

31 Dolly Rizvi, "Begum Akhtar ", dans Great Musicians of India (Bombay : I.B.H. Publishing Company, 1969), 4.

32 Ce poème favori de Dagh a d'abord été chanté par la chanteuse pakistanaise Iqbal Bano. 
« classique ». Grâce à sa formation et à son expérience antérieure elle avait la flexibilité personnelle et musicale nécessaire pour s'exprimer dans des contextes divers et pour répondre aux attentes d'auditoires différents. Cette sensibilité lui a permis de transformer les présentations intimes des salons, avec leur émotivité à la fois intense et souple, en un acte musical authentique imprégné de classicisme : le style du récital de musique légère. Lors de la présentation d'un de ses récitals, Begum Akhtar a déclaré : "Le ghazal et le style avec lequel on le chante constitue un des genres de la musique classique. Chanté d'une façon authentique, il a un impact profond, pourvue que l'on respecte le " bon goût" de la culture musicale. ${ }^{33}$ "

Quel est donc ce "bon goût " ? Les musiciens et les musicologues s'entendent pour dire qu'il comporte deux aspects principaux : l'un touche à l'usage compétent que l'on fait des différents ragas, l'autre consiste en un idiome de composition que l'on peut identifier en se référant aux traits stylistiques, aux écoles ou aux régions. Rattaché aussi à ces deux aspects est la légitimité du disciple qui doit être le descendant, pour ainsi dire, d'un maître reconnu. Parmi les chanteuses de musique classique légère, Begum Akhtar se distingue par son répertoire de raga et son habilité à composer spontanément dans ce genre. Du point de vue stylistique, sa musique est caractérisée par un mélange idiomatique de deux styles : celui, profondément expressif, du Purab, sa propre région, et celui, romantique et ornementé, du Punjab. L'authenticité de ce style « idiomatique » d'Akhtar est légitimée par son affiliation, en tant que disciple, non seulement à Ata Muhammad Khan, mais plus particulièrement au maître Abdul Wahid Khan, l'un des deux plus grands maîtres de musique classique de notre siècle.

Le fait que Akhtar a été disciple propre (ou gandaband) du célèbre maître Wahid Khan ${ }^{34}$ semble avoir d'avantage confirmer la légitimité de son art, ce que ne pouvaient lui apporter ses autres professeurs puisqu'ils ne formaient et n'accompagnaient que des courtisanes. Quant à la sorte d'association qu'elle a eue avec Abdul Wahid Khan, on ignore tout sauf qu'elle a dû avoir lieu après avoir quitté Calcutta et avant 1947, tout probablement à l'occasion de ses visites à Bombay. L'accompagnement que lui fournissait souvent le distingué joueur de sarangi, Shakoor Khan, a servi à maintenir le lien avec le grand maître dont il était le neveu ${ }^{35}$. Beaucoup plus tard, Begum Akhtar est redevenue disciple, cette fois du réputé Arvind Parikh, avec qui elle a étudié le sitar dans le style vocal de son grand professeur Vilayat $\mathrm{Khan}^{36}$. Stimulée par sa quête incessante

33Même en ourdou le terme est klassiki mausiqi, comme l'emploie Begum Akhtar dans son introduction ; voir le texte du disque Begum Akhtar Talks About, and Sings, the Ghazal (1975).

34Le terme gandaband (lié d'un fil sacré) représente le lien permanent entre le disciple et le maître.

35Misra, Music Makers of the Bhatkhande College, 76-77 ; Sharma, Musicians of India Past and Present, 144 ; Vasudev, « Ghazal Queen ». Abdul Wahid Khan habitait d'abord à Bombay mais, à partir de 1947, il a demeuré au Pakistan où il est mort en 1949.

36 Arvind Parikh la considérait comme une artiste trop élevée pour lui, mais elle tenait à devenir son disciple et le maître de ce dernier, Vilayat Khan, lui a ordonné de l'accepter ; Parikh, conversation avec Regula B. Qureshi (Bombay, 1994.) 
d'une formation classique, cette relation lui a valu également des conseils et l'appui personnel d'un membre éminent de l'élite musicale de Bombay.

Ce qui est étonnant, c'est que Begum Akhtar a continué à étudier et est devenue disciple même après avoir atteint le sommet de ses capacités artistiques et de sa popularité. Est-ce là un signe que cette artiste reconnaissait implicitement que sa légitimité et son expertise dépendaient de professeurs masculins ? Un incident révélateur, raconté par Arvind Parikh, suggère que c'était peut-être le cas et qu'une telle dépendance relevait de sa formation et de son expérience musicale. Lors de sa première leçon avec elle, il avait été étonné que Begum Akhtar lui tende son tamboura ${ }^{37}$ afin qu'il l'accorde, au lieu de réclamer elle-même le contrôle sur l'intonation de son instrument d'accompagnement, comme l'aurait fait tout soliste masculin. Elle lui a expliqué qu'elle n'avait jamais appris à le faire puisque son accompagnateur au sarangi s'en était toujours occupé et accordait lui-même son tamboura ${ }^{38}$.

On ne sait pas jusqu'à quel point les professeurs de Begum Akhtar ont contribué à sa formation classique et à ses compositions. Peut-être ont-ils composé de sa musique ; mais ils est possible aussi que les compositeurs nommés sur certains de ses enregistrements n'ont fait qu'effectuer des arrangements pour les ensembles de studio. Quoiqu'il en soit, la musique de Begum Akhtar que l'on entendait était perçue comme étant entièrement la sienne : " toujours sincère et édifiante ${ }^{39}$, sa musique était jouée " d'une manière typique qui est maintenant reconnue à travers l'Inde et le Pakistan comme étant son style individuel ${ }^{40}$. » En plus de se servir des ragas, deux traits musicaux contribuent directement à ce que le poète Faiz appelle « le grand sérieux de l'expression classique ${ }^{41}$. L'un est un mouvement rythmique relativement lent, même s'il est animé quelque peu par des ornements mélodiques agiles et qu'il est accéléré durant les interludes instrumentaux. L'autre consiste en une prédilection pour les longues durées : durée d'une note importante, d'une phrase ou même d'une pièce entière, le tout enrichi d'improvisations mélodiques. On a caractérisé ce style de "limpide, calme, et serein », mais ce qu'il suggère surtout c'est un certain recul conscient, une certaine nostalgie. Selon les paroles mêmes de son biographe de Lucknow, « son chant évoquait toujours les images de tout ce qui avait rendu célèbre et admirable la culture de Lucknow ${ }^{42}$. »

Le style des récitals de Begum Akhtar privilégiait ce qui avait toujours été au cœur même de son identité musicale : sa voix. La très grande souplesse et la tessiture étendue de sa voix lui permettaient de rendre toute une gamme d'ornements qui incorporait habilement le difficile passage d'un registre à l'autre, pierre d'achoppement pour bien des chanteurs. Elle en faisait un orne-

37 Cet instrument à cordes, qui fournit un accompaniment de bourdon, est joué soit par la chanteuse elle-même ou par un accompagnateur, surtout en concert.

38Parikh, conversation avec Regula B. Qureshi (Bombay, 1994).

39Selon le compositeur Murli Manohar Swarup ; voir le texte du disque Lost Horizons : Begum .4khtar-Ghazals (1977).

40Lutfullah Khan ; voir le texte du disque Begum Akhtar (1969).

41 Faiz ; voir le texte du disque Begum Akhtar in Memoriam : Kalam-e-Asatiza (1975).

42Misra, Great Masters of Hindustan Music, 174. 
ment des plus expressifs ${ }^{43}$. Par ailleurs, avec les années, la tessiture de sa voix est devenue plus grave et son timbre s'est rapproché de celui de la voix d'alto, mais sans perdre de sa beauté ni de son agilité.

Ce que Faiz a appelé figurativement « la mélancolie mielleuse de sa voix »4 était devenu, par l'entremise de ses récitals, la sonorité de l'expérience vécue, de la poésie vivante, de l'émotion et de l'intimité. Pour son public, son chant évoquait un passé féodal nostalgique ; mais sa voix conservait aussi son allure personnelle et représentait toute l'expérience esthétique et personnelle de Begum Akhtar. Si sa vie professonnelle lui imposait la solitude, en revanche elle cherchait toujours à établir un lien intime avec ses auditeurs. La musique était devenue pour elle l'occasion d'une échange réciproque d'affection avec son auditoire.

Durant ses tournées de récitals, et spécialement lors de ses séjours prolongés dans les grands centres, Begum Akhtar accueillait toujours, de sa manière généreuse, amis et amateurs de musique. Son ami G. N. Joshi, directeur de la musique classique à la compagnie EMI, se souvient de ses visites en soirée à l'hôtel Seagreen South à Bombay, accompagnée d'autres membres de la société musicale de Bombay ${ }^{45}$; à Delhi, c'était à l'hôtel Nirula que ses amis et ses admirateurs se réunissaient pour la voir. En tant que femme mariée, elle ne demeurait que rarement chez ses bienfaiteurs ; cependant, elle continuait d'accepter des engagements privés chez des aristocrates cultivés. Les soirées en compagnie d'un auditoire de connaisseurs choisis demeurait le contexte qui inspirait le plus Begum Akhtar à communiquer spontanément et chaleureusement par sa musique. Les réparties lancées occasionnellement à un auditeur particulier soulignaient la qualité personnelle et spontanée de son expérience musicale. Le seul enregistrement que nous avons d'un tel événement est celui qui a été réalisé dans la demeure du poète-mécène Jasdanwalla et qui nous démontre, brièvement mais précieusement, sa pratique de personnaliser sa musique en intégrant à ses récitals un aspect verbal aussi informel ${ }^{46}$.

Les auditeurs de Begum Akhtar, à leur tour, n'ont pas manqué de lui manifester leur affection. Ils se souviennent non seulement de son art mais aussi de sa personnalité. Pour eux, elle était une dame de Lucknow hautement placée, éminemment respectable, d'une grande maturitét ${ }^{47}$, mais une chanteuse professionnelle dont les grandes qualités artistiques et l'habileté à plaire par ses récitals leur faisaient apprécier davantage sa générosité et la gracieuseté chaleureuse de sa personnalité. "Chérissant grandement les enfants ${ }^{48}$, « elle était, pour de nombreux admirateurs et jeunes musiciens-aspirants, l'affectueuse ammi (mère, maman) ${ }^{49}$. "

43 Shakoor Khan, cité dans Vasudev, « Ghazal Queen »; et Joshi, Down Memory Lane, 68.

44Faiz Ahmad Faiz ; voir le texte du disque Begum Akhtar in Memoriam : Kalam-e-Asatiza (1975). 45 Joshi, Down Memory Lane, 70 ; Vasudev, "Ghazal Queen ".

46Voir le texte du disque Malika-E-Ghazal Begum Akhtar in Mehfil (1983) ; voir aussi Misra, Music Makers of Bhatkhande College ; idem, Great Masters of Hindustan Music ; Vasudev, " Ghazal Queen »; et Joshi, conversation avec Regula B. Qureshi (Bombay, 1988).

47Zia, conversation avec Regula B. Qureshi (Sudbury, Ontario, 1994).

48 Rizvi, « Begum Akhtar », 1.

49Misra, Great Masters of Hindustan Music, 180 ; voir aussi Surya, « Ammi », 46-47. 
Pourtant, il semble que Begum Akhtar demeurait vulnérable, car elle avait besoin qu'on l'appuie même lorsqu'elle était au sommet de sa renommée et " qu'elle baignait dans les adulations toujours croissantes des classes et des masses $^{50}$. » G. N. Joshi, le patron respecté pour qui elle enregistrait, raconte comment, en studio, un administrateur inculte de la compagnie EMI lui avait suggéré de chanter dans le style commun des chanteurs populaires ; ceci l'a tellement déconcertée qu'elle n'a pu continuer la session d'enregistrement. Pire encore, en apprenant par ouï-dire que Joshi avait critiqué son nouveau disque, elle est demeurée inconsolable jusqu'à ce qu'il l'assure de son éternelle admiration pour son $\operatorname{art}^{51}$. Malgré son admirable pondération et son « incomparable assurance sur la scène ${ }^{52}$, elle dépendait toujours énormément des hommes qui avaient créé et contrôlé sa carrière, voire sa vie entière. S'il est vrai, sur le plan personnel, que la situation de dépendance dans laquelle la mettait son mariage la libérait vis-à-vis d'autres hommes, sur le plan professionnel, elle continuait d'exiger l'appui des mécènes et d'autres musiciens.

Apparemment, Begum Akhtar n'a pas pu réaliser ce qui était clairement le mandat exceptionnel d'une chanteuse professionnelle de son calibre. Mais, quant aux raisons de son échec, on ne peut que spéculer sur le rôle qu'aurait joué une certaine insécurité de sa part, particulièrement vis-à-vis des musiciens. Elle avait été invitée à assumer la direction de la nouvelle chaire de «musique légère » au fameux Marris College of Music, à Lucknow ${ }^{53}$, mais les résultats de cette initiative n'ont pas été très heureux. De plus, son projet consistant à donner des cours chez elle n'a pas eu de succès non plus, même si « des amateurs de musique se bousculaient pour profiter de son enseignement ${ }^{54}$. Pour Susheela Misra, qui voulait étudier avec elle, Begum Akhtar passait trop de temps en tournée à l'extérieur de Lucknow et, d'ailleurs, elle «n'avait pas le tempérament qu'il fallait pour enseigner ${ }^{55}$. » Doit-on conclure alors qu'elle était incapable de passer à un poste d'autorité qui, par tradition, appartenait aux maîtres masculins avec qui toutes les femmes musiciennes acceptaient d'étudier?

Begum Akhtar a néanmoins prodigué un certain enseignement, bien que sur une base individuelle seulement et à très peu d'étudiantes, toutes des femmes provenant de la classe moyenne ${ }^{56}$. La seule qui a étudié avec elle d'une façon prolongée est Shanti Hiranand, qui a vécu avec elle pendant quelques années et qui l'accompagnait régulièrement dans ses récitals en tant qu'appui vocal, comme le font les disciples des maîtres classiques. Elle avait été clairement désignée pour lui succéder comme femme musicienne, mais, de manière signi-

50Misra, Great Masters of Hindustan Music, 180.

51 Ce disque, enregistré par quelqu'un d'autre que G. N. Joshi chez HMV, est probablement Lost Horizons. Les deux incidents sont rapportés dans Joshi, Down Memory Lane, 71-73.

52 Joshi, Down Memory Lane, 72 ; et Rizvi, «Beghum Akhtar », 1.

53Misra, Music Makers of the Bhatkhande College, 75. Le collège s'appelle Bhatkhande College depuis l'indépendance de l'Inde.

54Misra, Music Makers of the Bhatkhande College, 77.

55Misra, Great Masters of Hindustan Music, 180.

56Misra identifie Shanti Hiranand, Sipra Bose, Anjali Banerji, Rita Gangoli et Kanwal Siddhu, dans Musical Heritage of Lucknow (New Delhi : Harman Publishing, 1991), 38 ; voir aussi Surya, "Ammi », 46-47. 
ficative, pour Shanti Hiranand, comme pour les autres disciples de Begum Akhtar, celle-ci demeurait une ammi, une figure maternelle plutôt qu' une figure de maître. C'est dire qu'il s'agissait d'une relation de parenté et de solidarité et non d'une relation de domination et de soumission. Ceci est souligné par le refus, chez Begum Akhtar, d'un statut officiel. Au Bhatkhande College, par exemple, où elle a enseigné pendant une brève période de temps, la tradition voulait que l'on utilise certains titres, comme celui de " professeur », pour s'adresser aux maîtres. Dans un sens formel, le terme n'était pas exclusif aux hommes. Cependant, Begum Akhtar s'est refusée au titre professionnel, ce qui revient à dire qu'elle se distanciait du statut qui y était rattaché.

Est-ce à dire que même une artiste de concert et un professeur aussi respectable que Begum Akhtar est demeurée essentiellement victime des contraintes sociales traditionnelles qui avaient façonné son expérience de la vie ? D'une part, une dichotomie morale déchirait son identité de femme ; elle devait s'occuper de sa compétence professionnelle, mais sans négliger ses liens familiaux. D'autre part, il y avait la dépendance totale dans laquelle vivaient les femmes par rapport aux hommes : l'identité musicale de la femme n'était légitime que si elle était cautionnée par un professeur mâle, tout comme l'identité parentale n'était légitimisée que par la parenté masculine. Cette conjoncture aurait bien pu empêcher Begum Akhtar d'assumer elle-même le pouvoir légitimant des maitres.

Il y a cependant une autre façon de comprendre son choix de ne pas assumer la position de " maître ». C'est que le cercle d'étudiantes et d'admiratrices de Begum Akhtar était constitué uniquement de femmes de la classe moyenne avec qui elle entretenait des relations d'appui mutuel, exemptes de la notion de pouvoir, étant caractérisés par des sentiments de solidarité, d'intimité, d'entraide et de naturel. C'est un modèle que l'on retrouve traditionnellement dans les maisons où les femmes, vivant ensemble, sont essentiellement libres de mener leurs affaires domestiques d'une manière autonome. Ce qui rendait ce cercle de femmes unique, c'est que Begum Akhtar y apportait l'expérience assez exceptionnelle d'une femme issue d'une famille matrilinéaire. Des rencontres personnelles et des récits publiés démontrent que cette expérience permet plus de souplesse dans les relations, même au sein d'une famille, et une spontanéité d'expression et de dialogue tant musical que verbal ${ }^{57}$. Ceci est significatif, car c'est par ce moyen que les chanteuses professionnelles rejoignent leur auditoire. Il en découle que le succès de Begum Akhtar est largement dû à sa manière d'être, manière marquée par des qualités " féminines » qui la distinguaient clairement du comportement plus réservé et moins expressif des femmes issues de la classe moyenne à laquelle appartenait la famille de son mari.

$57 \mathrm{Y}$ compris des conversations et des histoires orales, des écrits littéraires tels Mirza Hadi Ruswa, Umrao Jan Ada (The Courtesan of Lucknow), trad. par Khushwant Singh et M. A. Husaini (Bombay : Orient Longmans, 1961) ; Abdul Halim Sharar, Lucknow : The Last Phase of an Oriental Culture, trad. et éd. par E. S. Harcourt and Fakhir Hussain (Londres : Paul Elek, 1975); des recherches telles celles de Veena Talwar Oldenburg, The Making of Colonial Lucknow 1857-1877 (Princeton : Princeton University Press, 1984) et « Lifestyle as Resistance : The Case of the Courtesans of Lucknow », Feminist Studies $16, \mathrm{n}^{\circ} 2(1990): 259-88$. 
Toutes significatives qu'elles soient, pourtant, il serait illusoire d'oublier que la rencontre de relations féminines, pour être acceptée, avait besoin d'une légitimité masculine, car, après tout, cette rencontre n'aurait pas été possible si Begum Akhtar n'avait pas assumé délibérement l'identité d'une épouse, et ce à l'exclusion de toute autre identité, surtout celle d'une chanteuse. Elle a pris le nom de son mari non seulement dans la vie civile mais aussi dans la vie professionnelle : lorsqu'elle a recommencé à chanter, c'est sous le nom de "Akhter Abbasi ${ }^{58}$. Durant toute sa carrière de concertiste, c'est sa légitimité de femme mariée qui lui a permis de se tailler une place unique parmi l'élite urbaine et les amateurs de musique classique de la classe moyenne, même si, d'elle-même, elle était la plus attrayante de toutes les musiciennes.

Les admirateurs et les biographes de Begum Akhtar ont mis en valeur la maturité de sa personnalité en reconstituant sa vie musicale à la lumière des dernières années de sa carrière et en laissant de côté les aspects négatifs de son état social. Leur initiative est singulièrement conforme à l'objectif culturel national qui consiste à transformer la musique privée et féodale en art public et national. En effet, la transformation de cette musique à caractère sensuel et intime suppose également qu'on redéfinisse son compositeur, ce qui demande un effort de collaboration faisant intervenir la relation entre l'artiste, ses bienfaiteurs et aussi son public. Begum Akhtar a non seulement réussi cette transformation musicale, mais son habileté à toucher les femmes l'a liée personnellement à des familles de la classe élite, créant ainsi des liens d'amitié qui transcendaient les origines sociales. En même temps, la dépendance fondamentale des femmes vis-à-vis des hommes de leur famille demeurait intacte et facilitait même ce processus.

Finalement, un domaine, celui de la religion, donne un accès équitable au mérite et permet même à l'habileté musicale de devenir méritoire. Les récits et les biographies parlent de la dévotion religieuse de Begum Akhtar et de son expression « musico-poétique » lorsqu'elle chantait des hymnes islamiques ${ }^{59}$. Puisque sa famille commémorait annuellement le rituel Shi'a Muharram, c'était toujours une occasion pour elle de devenir l'interprète célèbre des longues et émouvantes lamentations qu'on y chantait. À l'instar d'autres musiciens, elle a été attirée par le soufisme et ses interprétations mystiques des poèmes d'amour qu'elle chantait ${ }^{60}$. Son pèlerinage à La Mecque en 1969 sera l'occasion pour elle d'enregistrer une série d'hymnes qui ont été radiodiffusés à maintes reprises lors des festivals musulmans ${ }^{61}$. Certes, comme les croyances islamiques excluent les hymnes du domaine musical, ils ne « comptaient " pas au point de vue musical et ne pouvaient donc pas faire partie du répertoire de concert de Begum Akhtar. Par ailleurs, grâce à l' anonymat des enregistrements, ses inter-

58Misra, Music Makers of the Bhatkhande College, 77. Prendre le nom de son mari n'avait jamais été une tradition musulmane indienne, mais, grâce à l'influence anglaise, c'est maintenant devenu la norme.

59Zia, conversation avec Regula B. Qureshi (Sudbury Ontario, 1994). Zia cite le regretté Qudrat Ali, qui était un ami de la famille Abbasi ; voir aussi Rizvi, « Begum Akhtar », 5.

60 Joshi, Down Melody Lane, 71 ; Misra, Musical Heritage of Lucknow, 55.

61 Rizvi, « Begum Akhtar », 5 ; Joshi, Down Memory Lane, 71-72. 
prétations constituaient un témoignage audible de l'intégrité personnelle qu'on lui connaissait, et contribuaient ainsi au respect qu'on lui portait.

En quoi consiste alors l'héritage musical de Begum Akhtar ? Franchissant toutes frontières, tant musicales que sociales, elle a atteint un niveau d'autorité culturelle remarquable qui englobait à la fois la musique et la poésie, la musique artistique et la musique de divertissement, la musique de salon et la musique de concert. Elle plaisait musicalement aux hommes mais affirmait en même temps une autorité musicale indépendante de la gent masculine. De tout ceci émerge une voix d'une complexité musicale particulièrement significative dont la résonnance parvient du domaine féminin mais transcende les limites imposées par les genres.

Aujourd'hui, la voix de Begum Akhtar revit à travers d'autres femmes. Parmi ses disciples, Shanti Hiranand a réussi à reprendre presque en entier le répertoire de son professeur, comme on peut le constater par ses enregistrements ${ }^{62}$. Plus significatif encore, par son enseignement à l' Académie nationale Bharatiya Kala Kendra de Delhi, elle transmet aux femmes l'héritage laissé par Begum Akhtar. Le style expansif et plein de sentiments qui nourrissait la créativité musicale de cette grande chanteuse revit également à travers la voix de la célèbre Shobha Gurtu, une de ses disciples qui a reçu une formation professionnelle semblable à la sienne ${ }^{63}$. Dans le Pakistan voisin, une autre chanteuse professionnelle extraordinaire, Farida Khanam, s'est remise à interpréter ses chansons.

Mais le plus impressionnant de tout est que la musique de Begum Akhtar a été préservée grâce à ses multiples enregistrements : depuis ses premières chansons de trois minutes jusqu'aux longues pièces de récital de ses dernières années. Ces pièces continuent d'être disséminées, consommées et savourées, comme le sont " la mémoire et l'arome de son chant ", toujours plein de fraîcheur, même après un quart de siècle ${ }^{64}$. La musique de Begum Akhtar, en harmonie avec la vie musicale indienne passée et présente, demeure profondément appréciée parce qu'elle réussit à amalgamer, d'une façon singulière, l'expérience et la représentation musicale à travers la voix et la personnalité d'une femme remarquable.

Appendice : Enregistrements de Begum Akhtar (disques 33 1/3 tours sauf avis contraire; par ordre chronologique d'enregistrement)

Ghazals of Begum Akhtar. Megaphone (Calcutta) JNLX-1006. S.d.

Great Hits of Begum Akhtar. Megaphone (Calcutta) HMV EDLP-2725. 1963. Notes sur la pochette du disque.

Begum Akhtar : Thumris and Dadras. HMV (Inde) ECLP-2374. Enregistré en 1968. Notes.

62Le répertoire de la musique de Begum Akhtar chanté par Shanti Hiranand a été enregistré au Pakistan : 4 cassettes, Shalimar Recording Co. (Islamabad) LC 0031, 0032, 0033, 0034.

63 Après la mort de Begum Akhtar, son mari Ishtiaq Abbasi a été ému aux larmes par le chant de Shobha Gurtu ; voir Misra, Music Makers of the Bhatkhande College, 77.

64 Joshi, Down Memory Lane, 68. 
Begum Akhtar Sings Ghalib : A Ghalib Centenary Production. HMV (Inde) ECSD-2399 (stéréo). Enregistré en 1968. Notes par Firaq Gorakhpuri.

Begum Akhtar. HMV (Pakistan) LKDA-20003. 1969. Enregistré durant une courte visite au Pakistan. Notes par Lutfullah Khan.

Begum Akhtar : Ghazals. HMV ECSD-2486 (stéréo). 1971. Arrangements et direction musicale : Khayyam. Notes.

Lost Horizons : Begum Akhtar - Ghazals. HMV (India) ECSD-2776 (stéréo). 1977 (enregistré en 1974). Direction musicale : Murli Manohar Swarup. Derniers enregistrements de Begum Akhtar réalisés aux studios de la HMV à Bombay. Notes par G. N. Joshi.

Begum Akhtar Talks About, and Sings, the Ghazal. Polydor (Inde) 2392-837. 1975 (date de l'enregistrement inconnue). Disque gravé à partir d'enregistrements de la All India Radio. Le disque comprend une présentation de chaque ghazal par Begum Akhtar.

Begum Akhtar in Memoriam : Kalam-e-Asatiza. HMV ECSD-2741 (aussi disponible sous forme de cassette : HMV (Pakistan) TC-CKDA-10010). 1975 (date de l'enregistrement inconnue). Arrangements et direction musicale : Khayyam. Notes par Faiz Ahmad Faiz.

Ghazals Forever : Begum Akhtar. HMV (Inde) 6TCS 04B 5012 (cassette ; stéréo). 1979. Arrangements et direction musicale : Khayyam.

Malika-E-Ghazal Begum Akhtar, In Mehfil. Music India 2393925 (aussi disponible sous forme de cassette : Music India 3227-712). 1983 (date de l'enregistrement inconnue). Extrait de la collection de disques de la famille Jasdanwalla. Notes.

Begum Akhtar : Thumris, Sawan, Ghazals. Music India 2393 924. 1983. Disque gavé à partir d'enregistrements de la All India Radio. Contient des photos (gracieuseté de Begum Shamim Bano, la sœur de Begum Akhtar).

Begum Akhtar : Thumri, Dadra, Poorvi. HMV (Inde) ECLP-2977. 1984 (date de l'enregistrement inconnue).

\section{Résumé}

L'article situe la vie de musicienne d'une grande chanteuse dont la carrière va du milieu féodal et colonial des cours et des salons jusqu'aux scènes fréquentées par la classe moyenne urbaine d'une Inde indépendante. On y retrouve la question habituellement problématique de l'identité sociale de cette musicienne héréditaire, qui est le corollaire inévitable de l'association entre hommes et interprètes, mais aussi la condition essentielle à l'apprentissage et à la pratique de l'art de la musique. Comment Begum Akhtar a rapidement acquis une réputation en musique grâce à l'appui des nobles, comment elle est « passée » dans le monde respectable du mariage, et comment elle a redéfini son art et sa personne, émergeant comme l'idole nationale de la culture musicale de cour de l'Inde - c'est seulement dans les marges que l'on peut voir tous ces aspects, et ce par le biais d'un kaléidoscope de sources positionnées et de l'expression musicale stylisée d'une artiste dépendante des hommes mais qui a aussi créé des liens musicaux avec des femmes (incluant l'auteure). 